\title{
Formation and Dissipation of Viscous Disks around Be Stars
}

\author{
A.T. Okazaki \\ Faculty of Engineering, Hokkai-Gakuen University, Toyohira-ku, \\ Sapporo 062-8605, Japan
}

\begin{abstract}
Based on the viscous decretion disk model for Be stars, we study the effect of the torque from the star on the Be star disk, using a 3-D smoothed particle hydrodynamics code and turning on and off the mass ejection from the star. We find that, after the mass ejection is shut off, the innermost part of the disk begins to accrete and the accreting part gradually propagates outward. We also find that the Be disk in misaligned binaries precesses in a retrograde sense when no mass is supplied from the star. If the mass supply is resumed for such a disk, an inner disk is formed with its rotation axis misaligned with that of the outer disk. By the interaction between the inner and outer disks, the outer disk rapidly changes its direction to that of the inner disk.
\end{abstract}

\section{Introduction}

Be stars are rapidly-rotating, non-supergiant early-type stars which have equatorial disks at least on one occasion. They can change themselves to B stars and back to Be stars again by losing the disk and then reforming it. Although there is no widely-accepted model for Be star disks, the viscous decretion disk model by Lee, Saio, \& Osaki (1991) explains many of the observed features and thus seems promising. The model assumes that the star can eject gaseous particles with the Keplerian velocity at the stellar equator. The ejected particles then gradually move outward by viscosity and form a disk.

In this model, the angular momentum is given by the central star and transported outward by viscosity (see also reviews by Townsend and Owocki, this proceedings). Such a disk cannot be steady. The disk grows when the star exerts a positive torque on it (Okazaki et al. 2002), whereas it is expected to decay when no/negative torque is exerted. In this paper, we study the effect of intermittent mass supply from the star on disk evolution.

\section{Numerical Model}

Simulations presented here were performed with a 3-D smoothed particle hydrodynamics (SPH) code based on a version originally developed by Benz (1990). The smoothing length was variable in time and space, and the SPH equations were integrated with individual timesteps for each particles (Bate, Bonnell, \& Price 1995). The artifificial viscosity parameters varied with time and space so as to keep Shakura-Sunyaev viscosity parameter $\alpha_{\mathrm{SS}}$ constant $\left(\alpha_{\mathrm{SS}}=0.1\right.$ in the simulations shown below). To model the mass ejection from the Be star, we 

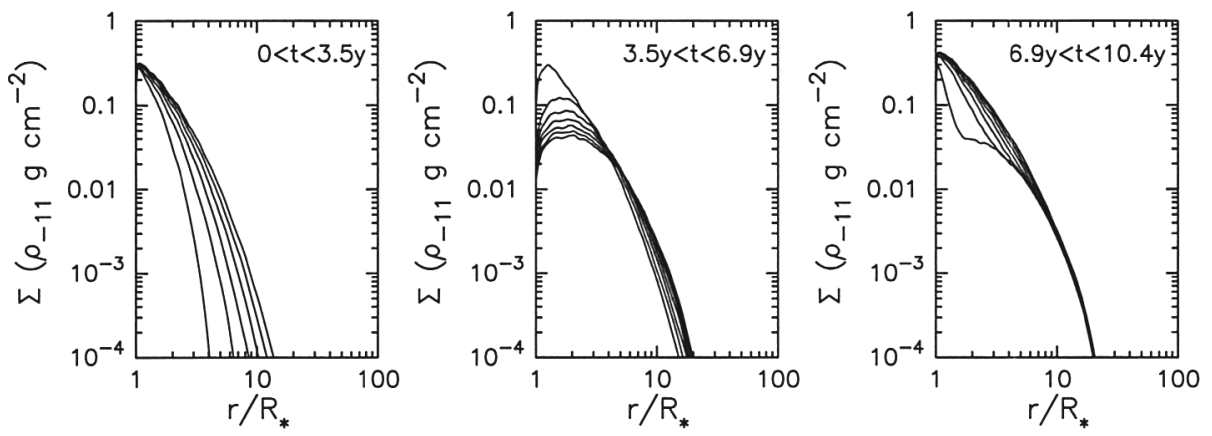

Figure 1. Surface density evolution of the viscous disk around an isolated Be star for $\alpha_{\mathrm{SS}}=0.1$. The mass supply rate from the star is constant for $t \lesssim 3.5 \mathrm{yr}$ (left panel), then set to zero for $3.5 \mathrm{yr} \lesssim t \lesssim 6.9 \mathrm{yr}$ (middle panel), and resumed at the same rate as for $t \lesssim 3.5 \mathrm{yr}$ (right panel). The surface density is measured in units of $\rho_{-11} \mathrm{~g} \mathrm{~cm}^{-2}$, where $\rho_{-11}$ is the highest local density at $t=1 \mathrm{yr}$ normalized by $10^{-11} \mathrm{~g} \mathrm{~cm}^{-3}$, a typical value for Be stars. The time interval between adjacent contours is $0.5 \mathrm{yr}$.

injected isothermal particles rotating at the Keplerian angular velocity at the stellar equatorial region into a radius just outside the stellar surface. We took a B0V star with $M_{*}=18 \mathrm{M}_{\odot}, R_{*}=8 \mathrm{R}_{\odot}$ and $T_{\text {eff }}=26000 \mathrm{~K}$ as the Be star. The Be disk was assumed to be isothermal at the temperature of $1 / 2 T_{\text {eff }}$. In binary simulations, we adopted a $1.4 \mathrm{M}_{\odot}$ neutron star as the secondary with an orbital period of $24.3 \mathrm{~d}$ (semimajor axis $a \sim 12 R_{*}$ ) and an orbital eccentricity of 0.34 , which were taken from parameters for $4 \mathrm{U} 0115+63$, one of the best studied $\mathrm{Be} / \mathrm{X}$-ray binaries. For the details of the model, see Okazaki et al. (2002).

\section{Disk Evolution around Isolated Be Stars}

Figure 1 shows that the evolution of the surface density of the viscous disk around an isolated $\mathrm{Be}$ star. In order to study the effect of the intermittent mass supply from the star, the mass injection rate into the inner disk radius was kept constant for $t \lesssim 3.5 \mathrm{yr}$ (left panel), then set to zero for $3.5 \lesssim t \lesssim 6.9 \mathrm{yr}$ (middle panel), and resumed at the same level as for the first $3.5 \mathrm{yr}$ (right panel). The middle panel of Figure 1 shows that, if no mass is supplied from the star, the accretion begins at the innermost region and the accreting region gradually propagates outward. Outside the accreting region, the disk diffuses outward by viscosity. Such a disk will be observed as a broad ring with the peak density decreasing with time.

When the mass supply from the star is resumed, the reformation of the inner disk begins. For a period less than a year (for $\alpha_{\mathrm{SS}}=0.1$ ), the inner disk has a much steeper density distribution than the outer disk does. Such a "twocomponent" disk will temporarily exhibit two sets of double-peaked line profiles, like those seen in X Per (Tarasov \& Riche 1995; Clark et al. 2001). 

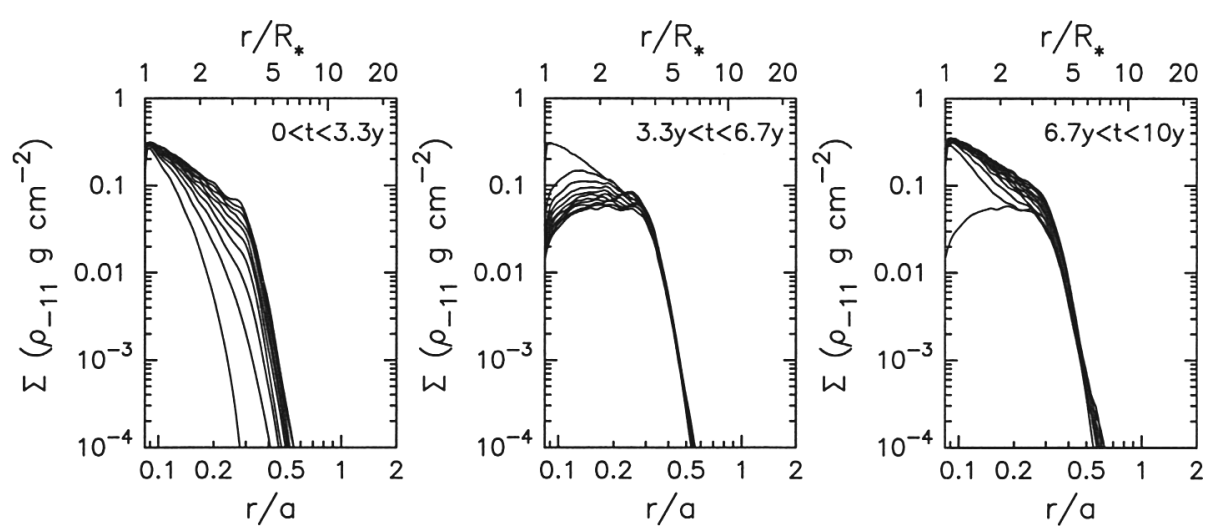

Figure 2. Surface density evolution of the viscous disk with $\alpha_{\mathrm{SS}}=0.1$ around a Be star in a binary with a neutron star orbiting at $P_{\text {orb }}=24.3 \mathrm{~d}$ and $e=0.34$. The mass supply rate from the star is constant for $t \leq 50 P_{\text {orb }}(\sim 3.3 \mathrm{yr})$ (left panel), zero for $50 P_{\text {orb }}<t \leq 100 P_{\text {orb }}$ (middle panel), and resumed at the same level as for $t \leq 50 P_{\text {orb }}$ (right panel). The time interval between adjacent contours is $5 P_{\text {orb }}(\sim 1 / 3 \mathrm{yr})$.

\section{Disk Evolution around Binary Be Stars}

In binary Be stars, the disk is expected to be truncated by the tidal/resonant interaction with the companion. The truncation of the $\mathrm{Be}$ disks in $\mathrm{Be} / \mathrm{X}$-ray binaries has been shown both observationally (Reig, Fabregat, \& Coe 1997; Zamanov et al. 2001) and theoretically (Negueruela \& Okazaki 2001; Okazaki \& Negueruela 2001; Okazaki et al. 2002). Below we separately describe the effect of the intermittent mass supply on the disk evolution under the influence of companion in coplanar systems and misaligned systems. At $t=0$, the neutron star companion is at apastron, which is in the $+x$-direction. The binary orbital plane is on the $x-y$ plane.

\subsection{Coplanar Disks}

Figure 2 shows the surface density evolution of a coplanar disk with $\alpha_{\mathrm{SS}}=0.1$. The mass injection rate into the inner disk radius was kept constant for $t \leq$ $50 P_{\text {orb }}(\sim 3.3 \mathrm{yr})$ (left panel), then set to zero for $50 P_{\text {orb }}<t \leq 100 P_{\text {orb }}$ (middle panel), and resumed at the same level as for the first $50 P_{\text {orb }}$ (right panel).

The effect of resonant truncation on the disk evolution is clearly seen in Figure 2. When the mass is supplied from the central star, the surface density of the disk increases more rapidly than for isolated Be stars. After the mass supply is shut off, the accretion in the inner region occurs as in disks around isolated Be stars, while the outward diffusion beyond the truncation radius is prevented by the resonant interaction with the companion. When the mass supply is resumed, the reformation of the inner disk region begins, temporarily showing a steep density profile near the star. 

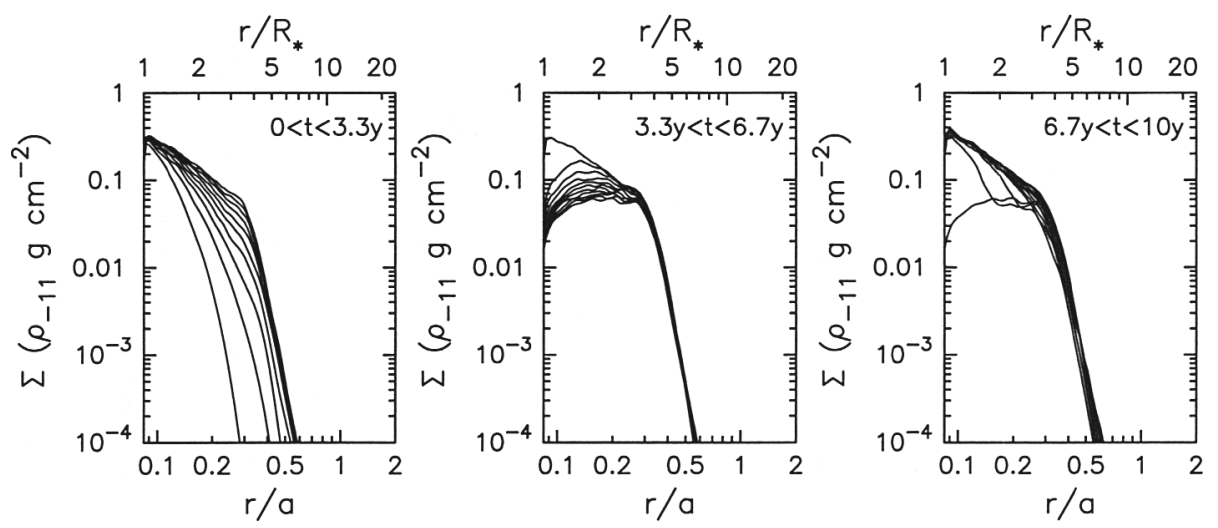

Figure 3. Same as Figure 2, but for a disk initially misaligned by $30^{\circ}$ about the $y$-axis.

\subsection{Misaligned Disks}

Figure 3 shows the effect of the intermittent mass supply on the surface denisty evolution of the disk in a system in which the rotation axis of the Be star is misaligned with that of the binary orbit. The angle of misalignement was initially $30^{\circ}$ about the $y$-axis. In Figure 3 , the radial coordinate is measured along the mean mid-plane of the tilted disk and the density is integrated along the direction perpendicular to it. It is immediately noted that the surface density evolution in the misaligned disk, when averaged in the azimuthal direction, is similar to that in coplanar disks.

However, taking a closer look at the disk structure, we noticed that there is a big difference between the evolution of misaligned disks and coplanar disks. For misaligned disks, the tidal precession can occur and the intermittent mass supply causes a much more complicated effect on the disk evolution than in coplanar systems. For $t \leq 50 P_{\text {orb }}(\sim 3.3 \mathrm{yr})$, when particles were supplied around the stellar equator at a constant rate, the tilt angle decreases gradually with the azimuth of the tilt changing little. When the mass supply was shut off at $t=50 P_{\text {orb }}$, the disk lost the torque from the Be star and began to precess in a retrograde sense. The precession rate during this period ( $\left.50 P_{\text {orb }} \lesssim t \lesssim 100 P_{\text {orb }}\right)$ was about $\sim 0.08 \Omega_{\text {orb }}$ with $\Omega_{\text {orb }}$ being the mean orbital frequency of the binary, which is in reasonable agreement with the analytical precession rate modified to include the effect of the orbital eccentricity (Bate et al. 2000 for inclusion of the effect of orbital eccentricity to the formula by Papaloizou \& Terquem 1995). By $t \sim 100 P_{\text {orb }}$, the disk has precessed through about $180^{\circ}$.

After the mass supply was resumed at $t=100 P_{\text {orb }}$, the disk structure changed drastically $\left(100 P_{\text {orb }} \lesssim t \lesssim 120 P_{\text {orb }}\right)$. In order to show what has happened on the disk, we present several snapshots of the disk covering this period in Figure 4. Each panel shows the view along the $y$-axis. From the first panel $\left(t \sim 101 P_{\text {orb }}\right)$, we note that an inner disk is newly formed around the stellar equator, the azimuth of which is different from that of the older, outer disk by about $180^{\circ}$. As the inner disk grew, the interaction with the outer disk via sound 

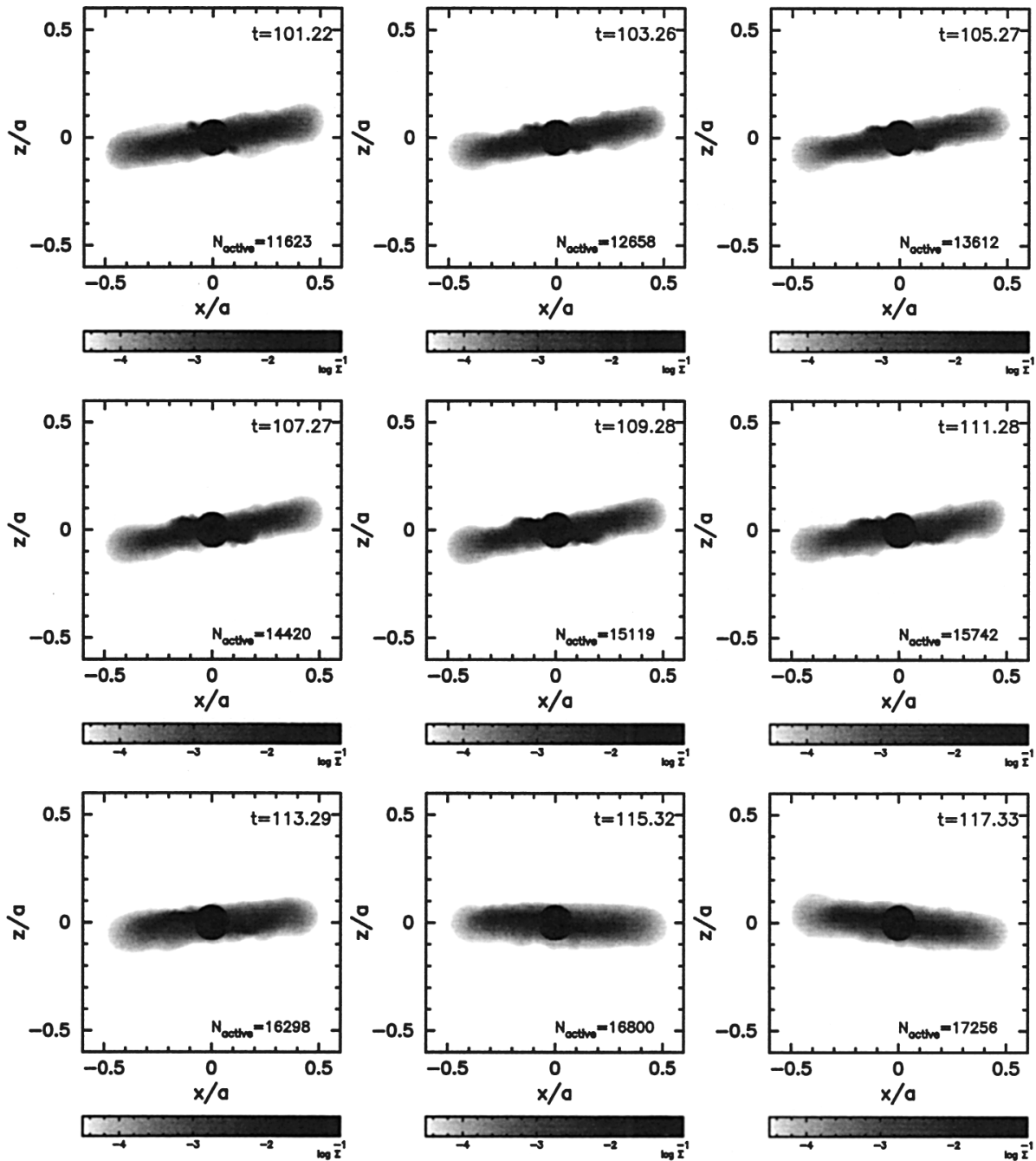

Figure 4. Snapshots of the disk with $\alpha_{\mathrm{SS}}=0.1$ during the period of a drastic change in the structure after the mass supply from the star was resumed. Epochs are chosen for illustrative purpose. Each panel shows the logarithm of the column density along the line-of-sight. The dark spot at the origin is the Be star. Annotated at the bottom of each panel is the number of SPH particles, $N_{\text {active }}$. The unit of time is $P_{\text {orb }}(=24.3 \mathrm{~d})$. 
wave propagation became stronger, which rapidly changed the tilt of the outer disk toward that of the inner disk $\left(105 P_{\text {orb }} \lesssim t \lesssim 115 P_{\text {orb }}\right)$.

\section{Conclusions}

Based on the viscous decretion disk model for Be stars, we have studied the effect of intermittent mass supply from the central star on the disk evolution, using a 3-D SPH code. We have found that, when the mass supply is shut off in isolated Be stars, accretion begins in the innermost part of the disk, and the accreting region gradually propagates outward, making a cavity around the star. Outside the accreting region, gaseous particles diffuse outward by viscosity. When the mass supply from the star is resumed, the inner disk is rebuilt up and the Be star may temporarily exhibit two sets of double-peaked line profiles, like those observed in X Per.

In coplanar binaries, in which the rotation axis of the Be star is aligned with that of the binary orbit, the effect of the intermittent mass supply on the disk evolution is similar to that in isolated Be stars, except that the outward diffusion is prevented by the resonant interaction with the companion.

In misaligned binaries, in which the rotation axis of the Be star is misaligned with that of the binary orbit, an interesting feature has emerged. The disk precesses little when the mass is supplied continuously from the star, whereas it precesses in a retrograde sense when no mass is supplied. Therefore, if the mass supply is resumed after a pause, the newly formed inner disk is inclined to the older outer disk. As the inner disk grows, the interaction with the outer disk becomes stronger and the outer disk rapidly changes its orientation toward that of the inner disk.

\section{References}

Bate, M.R., Bonnell, I.A., Clarke, C.J., Lubow, S.H., Ogilvie, G.I., Pringle, J.E., Tout, C.A. 2000, MNRAS 317, 773

Bate, M.R., Bonnell, I.A., Price, N.M. 1995, MNRAS 285, 33

Clark, J.S., Tarasov, A.E., Okazaki, A.T., Roche, P., Lyuty, V.M. 2001, MNRAS 380, 615

Lee, U., Saio, H., Osaki, Y. 1991, MNRAS250, 432

Negueruela, I., Okazaki, A.T. 2001, A\&A 369, 108

Okazaki, A.T., Bate, M.R., Ogilvie, G.I., Pringle, J.E. 2002, MNRAS 377, 967

Okazaki, A.T., Negueruela, I. 2001, A\&A 377, 161

Papaloizou, J.C.B., Terquem, C. 1995, MNRAS 274, 987

Reig, P., Fabregat, J., Coe, M.J. 1997, A\&A 322, 193

Tarasov, A.E., Roche, P. 1995, MNRAS 276, L19

Zamanov, R.K., Reig, P., Mart, J., Coe, M.J., Fabregat, J., Tomov, N.A., Valchev, T. 2001, A\&A 367, 884 